\title{
A NON INVASIVE METHOD FOR DIAGNOSIS AND ASSESSMENT OF PORTAL HYPERTENSION IN EGYPTIAN CIRRHOTIC PATIENTS USING PLASMA MALONDIALDEHYDE LEVEL
}

\section{By}

\author{
AHMED SAMIR ALLAM ${ }^{1}$, AYMAN GAMIL ANWAR ${ }^{1}$, RAMY SAMIR GHAIT ${ }^{1}$, \\ TAMER ABDEL RAHMAN ${ }^{2}$, WALAA AHMED YOUSRY KABIEL ${ }^{3}$, \\ HAYTHAM M. NASSER ${ }^{4}$ AND MOHAMED HASSANY ${ }^{5^{* *}}$ \\ Departments of Internal Medicine ${ }^{1}$, Tropical Medicine ${ }^{2}$, Clinical Pathology ${ }^{3}$ and \\ Radiology ${ }^{4}$, Faculty of Medicine, Ain Shams University, Cairo 11211, and \\ National Hepatology and Tropical Medicine Research Institute ${ }^{5}$, Cairo, Egypt \\ ( ${ }^{*}$ Correspondence: ahm82allam@gmail.com, ${ }^{* *}$ mohamadhassany@yahoo.com)
}

\begin{abstract}
Liver cirrhosis is a common disease affecting Egyptian patients. Complications of liver cirrhosis usually start once portal hypertension increases. To the authors' knowledge, there are no available non-invasive methods for assessment of the severity of portal hypertension. This study evaluated the value of plasma Malondialdehyde (MDA) - a lipid peroxide marker for oxidative stress, as a diagnostic biomarker, to assess severity of Portal Hypertension in Egyptian cirrhotic patients. It is a case-control study conducted on a total of 150 Egyptian patients divided into two groups. GI: 100 patients diagnosed as liver cirrhosis with esophageal varices proved by upper GIT endoscopy. GII: 50 normal controls. Serum MDA was measured by ELISA technique.

The results showed that MDA assay between the two groups revealed significant statistical difference between the two studied groups. Correlation between MDA and the clinical parameters in the cirrhotic group showed a significant positive correlation with ascites, Child Pugh score, varices grade, while there was no correlation with sex.
\end{abstract}

Keywords: Malondialdehyde; portal hypertension; liver cirrhosis.

\section{Introduction}

Portal hypertension is one of the most common complications of liver cirrhosis and one of the major causes of death in cirrhotic patients (Koga et al, 2019). Portal hypertension occurs as a result of the increase in the portal vein blood flow resistance caused by alteration in liver structure as well as alteration in blood structure (Parola and Robino, 2001).

Hepatic venous pressure gradient (HVPG) measurement is considered the gold standard method for the assessment of presence and severity of Portal Hypertension (PHT). However, it is an invasive tool and requires an experienced radiologist in its measurement (Groszmann et al, 2005).

Oxidative stress (OS) refers to a condition under which an organism or cell reactive oxygen species (ROS) are excessively produced and the antioxidant defense function is weakened, which causes big imbalance and damage to the organism cell (Catapano et al, 2000). Oxidative stress response acelerated liver fibrosis and also, initiates hepatic epithelioid malfunction, which is adjusted by the bioavailability of nitric oxide (NO) in the intrahepatic microcirculation (Rodriguez et al, 2007). End products of lipid peroxidetion have been used as biomarkers of the oxidative stress. These include malondialde hyde (MDA), isoprostanes and 2-propenal (acrolein). These compounds can be quantified in serum and urine and have the advantage of being relatively stable. Therefore, they can be used as an indirect measure of the oxidative stress (Raj et al, 2011)

Bajpai et al. (2017) described that the malonaldehyde (MDA) and the superoxide dismutase (SOD) were the most recognized predicative markers for the OS. MDA is the most representative indicator of OS in the body, while SOD is representative of the body's antioxidant system, and its function is to clear ROS. Also, Aziz et al. (2015) found that MDA levels significantly increased in 
human schistosomiasis and correlated significantly with hepatic fibrosis. This may confirm the role of LPO byproducts in schistosomiasis pathogenicity, and proposing malondialdehyde as a biomarker for schistosomiasis morbidity.

This study aimed to evaluate the value of plasma Malondialdehyde (MDA); a lipid peroxide marker for oxidative stress, as a diagnostic biomarker to assess severity of portal hypertension in the Egyptian cirrhotic patients.

\section{Materials and Methods}

The study was approved by the Research Ethics Committee of Ain Shams University reference number: 000017585. Also, informed written consent was obtained from each participant before enrolment in the study.

This is a case-control study conducted on a total of 150 Egyptian patients selected from Internal Medicine and Hepatology outpatient clinics and inpatient wards at Ain Shams University's Hospitals and National Hepatology and Tropical Medicine Research Institute in the period from May 2017 to June 2018. All procedures followed were in accordance with the ethical standards of the responsible Committee on Human Experimenttation (Institutional and National) and with the Helsinki Declaration of 1975, as revised in 2000.

This was a case control study; conducted on 150 patients classified into 2 groups, GI: 100 patients diagnosed as the liver cirrhosis with esophageal varices proved by upper GIT endoscopy and GII: 50 normal controls. MDA serum was measured by ELISA.

The excluded criteria of patients: Presence of bleeding esophageal varices, use of the vasoactive and antioxidant agents within a week, portal vein thrombosis, cardiac, renal or respiratory failure, hepatocellular carcinoma, abuse of alcohol and presence of active infection.

All patients were subjected to the full history taking, clinical examination, laboratory investigations including: fasting blood glucose, liver function tests (AST, ALT, prothr- ombin time, INR, serum albumin, total \& direct bilirubin), renal function tests (creatinine, urea, $\mathrm{Na}, \& \mathrm{~K}$ ), complete blood count (CBC) and measurement of serum MDA using ELISA technique.

Child-Pugh score which assessed bilirubin, albumin, INR, presence and severity of ascites and encephalopathy, was used to classify patients in class A, B or C. (Cholongitas et al, 2005)

Radiological examination: Abdominal ultrasound with measurement of portal vein (PV) caliber and PV duplex. Equipment used: Hitachi, EUB-5500, 2-5 MHz convex probe, (China, Mainland); the patients were examined in supine position with emphasis on liver by an experienced sonographist, who was blind to all biochemical characteristics of the participants.

Endoscopy: All patients underwent an upper gastrointestinal endoscopy after premedication with intravenous midazolam (2.5$7.5 \mathrm{mg}$ ), by video-endoscope after an overnight fast. During the upper gastrointestinal endoscopy, esophageal varices were graded as follow: 1- Small (Grade 1) minimally elevated veins above the esophageal mucosal surface, 2- Medium (Grade 2) tortuous veins occupying less than one-third of esophageal lumen and 3- Large (Grade 3): occupying more than one-third of esophageal lumen (De Franchis2005)

Serum MDA: Serum MDA was measured by Enzyme Linked Immunosorbent assay (ELISA) using Human MDA ELISA KIT by Glory Science Co., Ltd 2400 Veterans Blvd. Suite 16 - 101, Del Rio, TX 78840, USA.

Sample collection and preparation: Four $\mathrm{mL}$ of peripheral venous blood were collected under complete aseptic conditions into plain vacutainer tube and samples were clotted for 30 minutes before centrifulgation for 15 minutes at $1,000 \mathrm{xg}$. The freshly prepared serum was immediately assayed or stored in aliquots at $-20^{\circ} \mathrm{C}$ until used for assaying serum MDA (=Myo Bio Source Human MDA ELISA Kit Cat. No. MBS728071).

Assay procedure: 1. Wells for diluted cali- 
brators, blank and samples were determined. $50 \mu \mathrm{L}$ of calibrators, blank and samples were added into the appropriate wells, respecttively followed by $50 \mu \mathrm{L}$ of detection reagent A. Plate was shaken gently by microplate shaker, covered with a sealer and incubated for $1 \mathrm{hr}$ at $37^{\circ} \mathrm{C}$. 2. Solution in plate was aspirated and washed with $350 \mu \mathrm{L}$ of wash solution was added to each well using an auto washer. 3. A total of $100 \mu \mathrm{L}$ of detected reagent $\mathrm{B}$ working solution were added to each well, incubated for 30 minutes at $37^{\circ} \mathrm{C}$ after covered with plate sealer. 4 . Aspiration wash process was repeated five times as in step 2 . 5. A total of $90 \mu \mathrm{L}$ of substrate solution was added to each well and covered with a new plate sealer. After the incubation for 15-25 minutes at $37^{\circ} \mathrm{C}$ and protecting the plate from light, the liquid turned blue by addition of substrate solution. 6. A total of $50 \mu \mathrm{L}$ of stop solution was added to each well. Liquid turned yellow by adding stop solution. 7 . Micro plate reader was run and measured at $450 \mathrm{~nm}$ immediately. A standard curve was drawn on graph paper by plotting absorbance obtained from each standard on vertical (Y) axis against its concentration in $\mathrm{ng} / \mathrm{mL}$ on the horizontal axis. Absorbance value of each sample determined the corresponding MDA concentration from the standard curve. Original concentration was calculated by multiplying the dilution factor. Assay range was $7 \mathrm{ng} / \mathrm{mL}-40 \mathrm{ng} / \mathrm{mL}$.

Statistical analysis: Data were coded, tabulated and introduced to a PC using Statistical package for Social Science (SPSS 19.0.1 for windows; SPSS Inc, Chicago, IL). Analysis was conducted, using mean, standard deviation $( \pm \mathrm{SD})$ for quantitative data. These tests were used: Two tailed Student's t test when comparing between two means and paired sample t-test of significance was used when comparing between related samples. $\mathrm{P}$ value $<0.05$ was considered significant. Sensitivity, specificity, PPV, NPV and accuracy were calculated. Analysis of variance (ANOVA) compared among different times in same group in quantitative data.

\section{Results}

Ages ranged between 40 \& 67 years (53.4 \pm 5.813$)$, and the age of controls ranged between $43 \& 67(53.25 \pm 6.935)$, without significant difference between them with $\mathrm{P}=0.927$ (Tab. 1).

Table 1: Comparison between groups as regards ages

\begin{tabular}{|l|c|c|c|c|c|c|c|c|}
\hline Age & \multicolumn{3}{|c|}{ Cases } & \multicolumn{3}{c|}{ Controls } & T & P-value \\
\cline { 1 - 7 } Range & 40 & - & 67 & 43 & - & 67 & \multirow{2}{*}{0.092} & \multirow{2}{*}{0.927} \\
\cline { 1 - 5 } Mean \pm SD & 53.400 & \pm & 5.813 & 53.250 & \pm & 6.935 & & \\
\hline
\end{tabular}

Males were $98(65.3 \%)$ \& females were $52(34.7 \%)$ without significant difference $(\mathrm{P}=0.098)$.

Table 2: comparison between the two groups as regards sex.

\begin{tabular}{|l|c|c|c|c|c|c|c|c|}
\hline \multirow{2}{*}{ Sex } & \multicolumn{2}{|c|}{ Cases } & \multicolumn{2}{c|}{ Controls } & \multicolumn{3}{c|}{ Total } & \multicolumn{2}{c|}{ Chi-Square } \\
\cline { 2 - 10 } & No. & $\%$ & No. & $\%$ & No. & $\%$ & $\mathrm{X}^{2}$ & P-value \\
\hline Male & 64 & 64.00 & 34 & 68.00 & 98 & 65.3 & 0.098 & 0.754 \\
\hline
\end{tabular}

In cirrhotic patients $20(20 \%)$ were child A, 38(38\%) were child B \& 42 (42\%) were child $\mathrm{C}$. Esophageal varices in cirrhotic 28

\begin{tabular}{|l|c|c|}
\hline Child's Pugh score & No. & $\%$ \\
\hline Child A & 20 & 20.00 \\
\hline Child B & 38 & 38.00 \\
\hline Child C & 42 & 42.00 \\
\hline Total & 50 & 100.00 \\
\hline OES. V & No. & $\%$ \\
\hline \multicolumn{3}{|l|}{} \\
\hline Small & 28 & 28.00 \\
\hline Medium & 32 & 32.00 \\
\hline Large & 40 & 40.00 \\
\hline Total & 100 & 100.00 \\
\hline
\end{tabular}


MDA essay, in patients the MDA essay ranged between 300 and $1830 \mathrm{~nm}$ with mean level $934.860 \pm 481.574 \mathrm{~nm}$, while in controls MDA essay ranged between 160 and 310 with mean level $238.250 \pm 45.330 \mathrm{~nm}$, this revealed significant difference between the two groups with $\mathrm{P}<0.001$ (Fig. 1)
Correlation between MDA and clinical parameters in cirrhotic group showed a significant positive correlation with ascites $(\mathrm{p}<0.001)$, child Pugh score $(\mathrm{p}<0.001)$, varices grade $(\mathrm{p}<0.001)$ and PHG grade $(\mathrm{p}<0.001)$, but without correlation with sex (Tab. 4).

Table 4: correlation between MDA and clinical parameters in cirrhotic group.

\begin{tabular}{|c|c|c|c|c|c|c|c|}
\hline \multirow{2}{*}{\multicolumn{2}{|c|}{ Variables }} & \multicolumn{4}{|c|}{ MDA } & \multicolumn{2}{|c|}{ T-Test or ANOVA } \\
\hline & & \multirow{2}{*}{$\frac{\text { No. }}{64}$} & \multirow{2}{*}{$\begin{array}{c}\text { Mean } \\
906.656\end{array}$} & \multirow{2}{*}{$\begin{array}{l} \pm \\
\pm\end{array}$} & \multirow{2}{*}{$\frac{\text { SD }}{491.528}$} & \multirow{3}{*}{$\begin{array}{c}\mathrm{T} \text { or } \mathrm{F} \\
-0.548\end{array}$} & \multirow{3}{*}{$\begin{array}{c}\text { P-value } \\
0.586\end{array}$} \\
\hline \multirow{2}{*}{ Sex } & Male & & & & & & \\
\hline & Female & 36 & 985.000 & \pm & 473.004 & & \\
\hline \multirow{4}{*}{ Ascites } & Negative & 28 & 634.143 & \pm & 262.850 & \multirow{4}{*}{4.127} & \multirow{4}{*}{$<0.001 *$} \\
\hline & Mild & 25 & 1069.643 & \pm & 474.076 & & \\
\hline & Moderate & 27 & 911.071 & \pm & 447.270 & & \\
\hline & Marked & 20 & 1266.875 & \pm & 601.551 & & \\
\hline \multirow{3}{*}{ Child's } & Child A & 20 & 639.778 & \pm & 408.978 & \multirow{3}{*}{5.222} & \multirow{3}{*}{$<0.001 *$} \\
\hline & Child B & 38 & 831.000 & \pm & 384.385 & & \\
\hline & Child C & 42 & 1160.238 & \pm & 507.557 & & \\
\hline \multirow{3}{*}{ OES. V } & Small & 28 & 471.643 & \pm & 197.798 & \multirow{3}{*}{31.770} & \multirow{3}{*}{$<0.001 *$} \\
\hline & Medium & 32 & 828.750 & \pm & 368.259 & & \\
\hline & Large & 40 & 1344.000 & \pm & 347.084 & & \\
\hline \multirow{3}{*}{ PHG } & Negative & 20 & 438.300 & \pm & 194.522 & \multirow{3}{*}{15.248} & \multirow{3}{*}{$<0.001 *$} \\
\hline & Mild & 26 & 790.385 & \pm & 379.838 & & \\
\hline & Severe & 54 & 1188.333 & \pm & 430.568 & & \\
\hline
\end{tabular}

Correlating MDA with laboratory parameters showed significant statistical correlation with platelets $(\mathrm{p}<0.05)$, albumin $(\mathrm{p}<0.05)$ and INR $(\mathrm{p}<0.05)$, but without significant in age, TLC, HGB, Creatinine, ALT, AST and T. Bilirubin (Tab. 5).

Table 5: correlation between MDA and laboratory parameters.

\begin{tabular}{|l|c|c|}
\hline \multirow{2}{*}{ Correlations } & \multicolumn{2}{|c|}{ MDA } \\
\cline { 2 - 3 } & $\mathrm{R}$ & P-value \\
\hline TLC & -0.118 & 0.415 \\
\hline HGB & -0.083 & 0.566 \\
\hline Platelet & -0.345 & $0.014^{*}$ \\
\hline Creatinine & 0.158 & 0.272 \\
\hline ALT & -0.044 & 0.759 \\
\hline AST & 0.137 & 0.342 \\
\hline Albumin & -0.337 & $0.017^{*}$ \\
\hline T.bilrubin & 0.268 & 0.060 \\
\hline INR & 0.378 & $0.007^{*}$ \\
\hline
\end{tabular}

Assessing value of MDA in diagnosis of portal hypertension was found at best cut off value of MDA > 410. It was associated with $96 \%$ sensitivity \& $98 \%$ specificity in diagn- osis of portal hypertension. Positive predictive value was $97.5 \%$ and negative predictive value was $90.9 \%$. The test accuracy was 97.3\% (Tab. 6 \& Fig. 3).

Table 6: MDA in diagnosing portal hypertension in Egyptian cirrhotic patients.

\begin{tabular}{|c|c|c|c|}
\hline Sensitivity & Specificity & PPV & NPV \\
\hline 96 & 98 & 97.5 & 90.9 \\
\hline
\end{tabular}

\section{Discussion}

Portal hypertension $(\mathrm{PH})$ is considered one of the main complications of liver cirrhosis that leads to increase in incidence of ascites, variceal hemorrhage and decompensation. Thus, early $\mathrm{PH}$ diagnosis is the important 
key in the managing patients with cirrhosis (Bosch et al, 2008)

The guidelines recommend the diagnosis of PH by the measurement of hepatic venous pressure gradient (HVPG) which was an invasive procedure and is only available in specialized centers. Noninvasive markers could be an appropriate alternative, but none of the markers which had been investigated, so far, have shown satisfactory specificity and sensitivity results (De Franchis, 2008). The oxidative stress (OS) has been shown to increase the progression of liver fibrosis during the chronic liver injury (Parola and Robino, 2001).

The OS also leads to the hepatic endothelial dysfunction through modulating NO bioavailability in the intrahepatic microcirculation. Consequently, the increased oxidative stress is important for the development of portal hypertension in cirrhosis (Catapano et al, 2000).

The Malondialdehyde (MDA) is the chemical product that results from lipid peroxidation of polyunsaturated fatty acids. The amount of MDA in tissues can give an idea about the degree of lipid peroxidation; moreover, it can be used as a marker for oxidative stress (Jain et al, 2002).

In this study, value of MDA was measured in 150 subjects divided into two groups, the first group contains 100 patients suffers from liver cirrhosis complicated with portal hypertension while second group contained 50 healthy subjects. There was a statistically significant difference between both groups regarding the MDA level with $(\mathrm{P}<0.001)$ where the cases groups was higher than the control group. This result agreed with (KueiChuan et al, 2009) who also found that the MDA level in cases group was higher than that of control group which suggested that more oxidants stress and weaker antioxidants protection exist in cirrhotic patients than in control subjects. Also, the present result agreed with Sheng-Lan et al. (2015) who found that the MDA level in patients was higher than that of control ones.
In the present study, there was a positive significant correlation between MDA value and presence of ascites in cirrhotic patients.

This result agreed with Kuei-Chuan et al. (2009) who mentioned that the MDA was slightly higher in the patients with ascitesregardless its degree- than in the patients without ascites. However, in the present study, there was positive significant correlation between the MDA level and degree of ascites.

In the present study, there was a positive significant correlation $(\mathrm{P}<0.001)$ between MDA value and Child Pugh score of the cirrhotic patients where MDA level in Child C Pugh score was higher than MDA level in Child B Pugh score was also higher than MDA level in Child A Pugh score. These results agreed with Sheng-Lan et al. (2015) regarding correlation between MDA and Child Pugh score $(\mathrm{P}<0.001)$.

Also, the present results agreed with KueiChuan et al. (2009) who suggested that the plasma MDA levels might increase in the concordance with the progression of the severity of both the cirrhosis and fibrosis.

In this study, there was a positive significant correlation between the MDA level, the grade of esophageal varices and PHG with $\mathrm{p}$ values $<0.001$, which agreed with ShengLan et al. (2015) regarding the correlation between MDA value and esophageal varices grade $(\mathrm{p}<0.001)$.

In the present study, a positive significant correlation between MDA level and portal vein diameter $(\mathrm{p}<0.001)$ was found. ShengLan et al. (2015) reported that the variation of MDA level relate to the severity of portal hypertension as the vessel diameter width of venous system was positively related to the portal vein pressure in portal hypertension caused by cirrhosis, and the main vessel width represented the portal pressure to the some extent.

In the present study, there was positive significant correlation between MDA value and INR $(p<0.001)$ that reflected the presence of positive correlation between MDA 
value and the worsening of synthetic liver function due to the progression of liver cirrhosis, although the present study showed a negative correlation between MDA value and plasma albumin level ( $\mathrm{p}=0.017$ ).

In the present study, serum MDA best cut off value of $>410 \mathrm{ng} / \mathrm{ml}$ was associated with $96 \%$ sensitivity and $98 \%$ specificity in the diagnosing of portal hypertension. The positive predictive value was $97.5 \%$ and the negative predictive value was $90.9 \%$. The accuracy of the test was $97.3 \%$. These results agreed with Kuei-Chuan et al. (2009) who reported that a slightly higher cut off value of MDA $>426.5 \mathrm{ng} / \mathrm{ml}$ was associated with sensitivity $78.6 \%$ and specificity $100 \%$. Also, the present results agreed with ShengLan et al. (2015) who stated that a slightly higher cut off value of MDA $>426.5 \mathrm{ng} / \mathrm{ml}$ was associated with sensitivity $78.2 \%$ and specificity $86.2 \%$.

In the present study, the low cut value off can be explained by the difference of the etiology of cirrhosis which is mainly viral in the present study while alcoholic cirrhosis was predominant in the other two studies and also can be explained by the prevalence of the schistosomiasis infection among the Egyptian cirrhotic patients which markedly affected the severity of the portal hypertension.

In the present study, the limitations were small number of the participants. Also, prospective studies were required to compare between cirrhotic patients with portal hypertension, and cirrhotic patients with non-portal hypertension to elucidate whether the Malondialdehyde level was related either to the cirrhotic stage or the presence of portal hypertension.

\section{Conclusion}

Generally speaking, the portal hypertension is defined by the pathological increase in the pressure of the portal venous system, and cirrhosis is the commonest cause of the portal hypertension.

The outcome results showed that the MDA level was significantly higher in the cirrhotic patients with portal hypertension than in the healthy ones and directly correlated with the PVD, esophageal varices, PHG, Child Pugh score.

The MDA proved to be the marker for diagnosing the liver cirrhosis with the portal hypertension and also for assessing severity of portal hypertension in Egyptian patients.

Competing interests: Authors declared that they neither have conflict of interest nor received funds.

Authors' contributions: A.A.S., A.A.G., G. R.S., R. T.A \& H.M. conceived and planned the experiments. K.W.A.Y. contributed to sample preparation. N. H.M. performed the ultrasound to the patients.

All authors provided critical feedback and helped shape the research, analysis and manuscript. All authors read and approved the manuscript.

\section{References}

Aziz, IA, Yacoub, M, Rashid, L, Solieman, A, 2015: Malondialdehyde; Lipid peroxidation plasma biomarker correlated with hepatic fibrosis in human Schistosoma mansoni infection. Acta Parasitol. 60:735-42.

Bajpai, J, Prakash, V, Kant, S, Verma, AK, Srivastava, A, et al, 2017: Study of oxidative stress biomarkers in chronic obstructive pulmonary disease and their correlation with disease severity in north Indian population cohort. Lung India 34:324-9.

Bosch, J, Abraldes, JG, Berzigotti, A, et al, 2008: Portal hypertension and gastrointestinal bleeding. Seminars in liver dis. 28:3-25.

Catapano, AL, Maggi, FM, Tragni, E, et al, 2000: Low density lipoprotein oxidation, antioxidants, and atherosclerosis. Curr. Opin. Cardiol. 15:355-63.

Cholongitas, E, Papatheodoridis, GV, Vangeli, M, Terreni, N, Patch, D, et al, 2005: Systematic review: The model for end-stage liver disease-should it replace Child-Pugh's classification for assessing prognosis in cirrhosis? Alim. Pharmacol. Therap. 22:1079-89.

De Franchis, R, 2005: Evolving consensus in portal hypertension: Report of the Baveno IV consensus workshop on methodology of diagnosis and therapy in portal hypertension. J. Hepatol. 43:167-76 
De Franchis, R, Eisen, GM, Laine, L, et al, 2008: Esophageal capsule endoscopy for screening and surveillance of esophageal varices in patients with portal hypertension. Hepatology 47: 1595-603.

Groszmann, RJ, Garcia-Tsao, G, Bosch, J, et al, 2005: Beta-blockers to prevent gastroesophageal varices in patients with cirrhosis. New England J. Med. 353:2254-61.

Jain, SK, Pemberton, PW, Smith, AS, et al, 2002: Oxidative stress in chronic hepatitis C; not just a feature of late stage disease. J. Hepatol. 36:805-11.

Koga, A, Toda, K, Tatsushima, K, Matsuubayashi, S, Tamura, N, et al, 2019: Portal hypertension in prolonged anorexia nervosa with laxative abuse: A case report of three patients. Int. J. Eat. Disord. 52, 2:211-5.

Kuei-Chuan, L, Ying-Ying, Y, Wang, YW, et al, 2009: Increased plasma malondialdehyde in patients with viral cirrhosis and its relationships to plasma nitric oxide, endotoxin, and portal presure. Dig. Dis. Sci. 55:2077-85.

Parola, M, Robino, G, 2001: Oxidative stressrelated molecules and liver fibrosis. J. Hepatol. 35:297-306.

Raj, V, Ravi, J, Bell, LN, Ghabril, M, Kamendulis, L, Klaunig, JE, et al, 2011: Oxidative stress in chronic liver disease: relationship between peripheral and hepatic measurements. Am. J. Med. Sci. 4:314-7.

Rodriguez-Vilarrupla, A, Bosch, J, Garcia, J C, et al, 2007: Potential role of antioxidants in treatment of portal hypertension. J. Hepatol. 46: 193-7.

Sheng-Lan W, Xin, Y, Zhu, D, Zhang, DW, et al, 2015: Relevance of plasma malondialdehyde level \& the severity of portal hypertension in cirrhotic patients. Int. J. Clin. Exp. Med 8: 11007-13

Explanation of figures

Fig.. 1: Comparison between two groups as regards MDA essay.

Fig.. 2: Correlation between MDA and portal vein diameter.

Fig. 3: Diagnostic performance of MDA in diagnosing portal hypertension in Egyptian cirrhotic patients. 

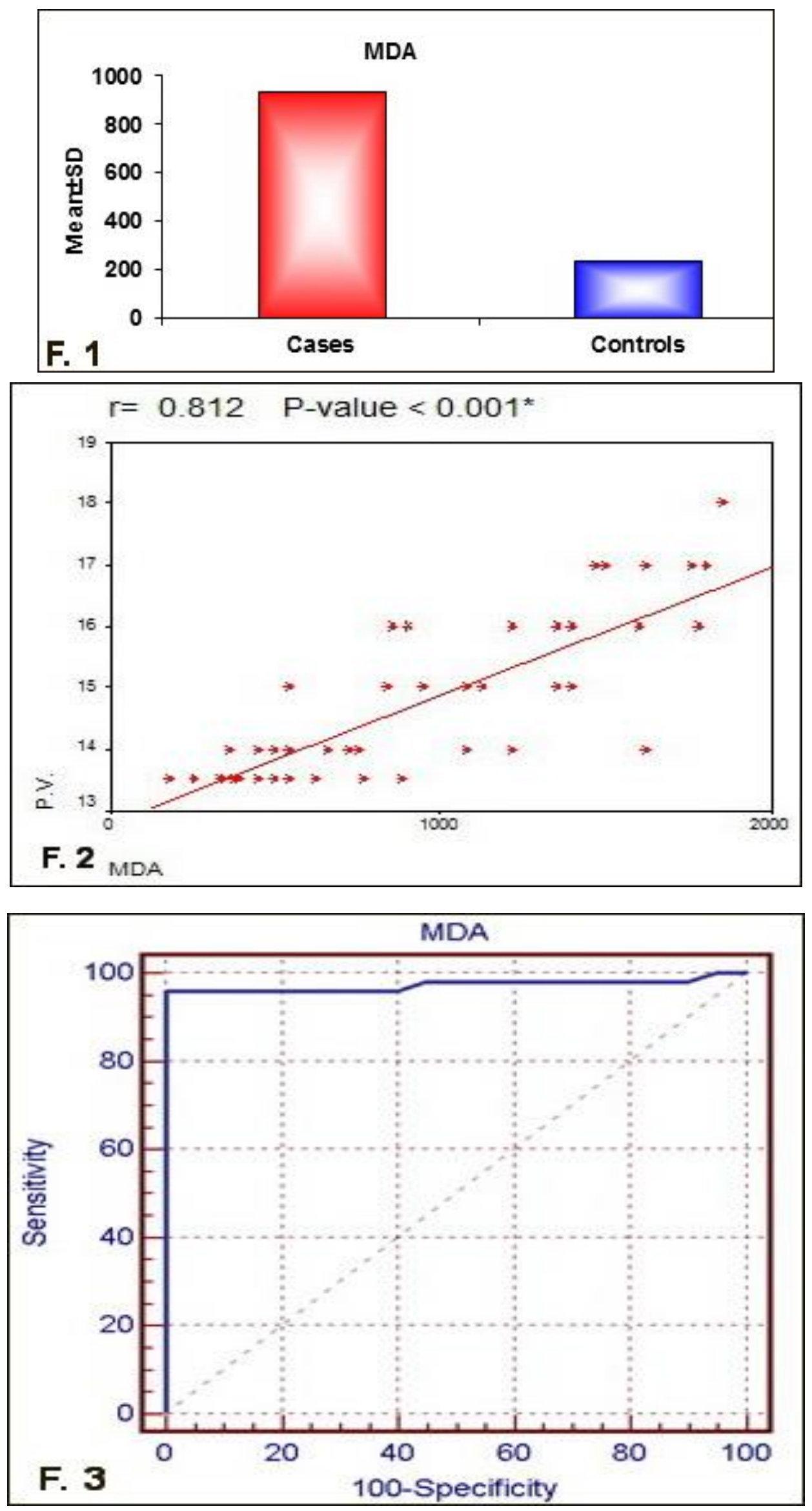